\title{
Results of ultrasound-assisted brace casting for adolescent idiopathic scoliosis
}

Edmond H. Lou ${ }^{1,2^{*}}$, Doug L. Hill ${ }^{1,2+}$, Andreas Donauer ${ }^{3 \dagger}$, Melissa Tilburn ${ }^{3 \dagger}$, Douglas Hedden ${ }^{1 \dagger}$ and Marc Moreau ${ }^{1 \dagger}$

\begin{abstract}
Background: Four factors have been reported to affect brace treatment outcome: (1) growth or curve based risk, (2) the in-brace correction, (3) the brace wear quantity, and (4) the brace wear quality. The quality of brace design affects the in-brace correction and comfort which indirectly affects the brace wear quantity and quality. This paper reported the immediate benefits and results on using ultrasound (US) to aid orthotists to design braces for the treatment of scoliosis.

Methods: Thirty-four AIS subjects participated in this study with 17 (2 males, 15 females) in the control group and 17 ( 2 males, 15 females) in the intervention (US) group. All participants were prescribed full time TLSO, constructed by either of the 2 orthotists in fabrication of spinal braces. For the control group, the Providence brace design system was adopted to design full time braces. For the intervention group, the custom standing Providence brace design system, plus a medical ultrasound system, a custom pressure measurement system and an in-house software were used to assist brace casting.
\end{abstract}

Results: In the control group, 8 of 17 (47\%) subjects needed a total of 11 brace adjustments after initial fabrication requiring a total of 28 in-brace radiographs. Three subjects (18\%) required a second adjustment. For the US group, only 1 subject (6\%) required adjustment. The total number of in-brace radiographs was 18 . The $p$ value of the chi-square for requiring brace adjustment was 0.006 which was a statistically significant difference between the two groups. In the intervention group, the immediate in-brace correction as measured from radiographs was $48 \pm 17 \%$, and in the control group the first and second in-brace correction was $33 \pm 19 \%$ and $40 \pm 20 \%$, respectively. The unpaired 2 sided Student's $t$ test of the in-brace correction was significantly different between the US and the first follow-up of the control group ( $p=0.02$ ), but was not significant after the second brace adjustment $(p=0.22)$.

Conclusions: The use of the 3D ultrasound system provided a radiation-free method to determine the optimum pressure level and location to assist brace design, resulting in decreased radiation exposure during follow-up brace evaluation, increased the in-brace correction, reduced the patients' visits to both brace adjustment and scoliosis clinics. However, the final outcomes could not be reported yet as some of patients are still under brace treatment.

Trial registration: NCT02996643, retrospectively registered in 16 December 2016

Keywords: Adolescent idiopathic scoliosis, 3D ultrasound imaging, Brace treatment, Brace design, Optimum brace pressure

\footnotetext{
* Correspondence: elou@ualberta.ca

${ }^{\dagger}$ Equal contributors

${ }^{1}$ Department of Surgery, University of Alberta, 6-110F, Clinical Science

Building, 8440-112 Street, Edmonton, Alberta T6G 2B7, Canada

${ }^{2}$ Department of Research and Innovation Development, Glenrose

Rehabilitation Hospital, Edmonton, Alberta T5G 0B7, Canada

Full list of author information is available at the end of the article
}

(c) The Author(s). 2017 Open Access This article is distributed under the terms of the Creative Commons Attribution 4.0 International License (http://creativecommons.org/licenses/by/4.0/), which permits unrestricted use, distribution, and reproduction in any medium, provided you give appropriate credit to the original author(s) and the source, provide a link to the Creative Commons license, and indicate if changes were made. The Creative Commons Public Domain Dedication waiver (http://creativecommons.org/publicdomain/zero/1.0/) applies to the data made available in this article, unless otherwise stated. 


\section{Background}

Adolescent idiopathic scoliosis (AIS) is a three-dimensional deformity of the spine associated with vertebral rotation due to an unknown cause. It is a chronic and a potentially progressive spinal deformity affecting $2-3 \%$ of the population [1]. Girls tend to progress more often than boys [2]. Although scoliosis is rarely life threatening, the long-term impact of untreated scoliosis is still controversial [3-8]. Patients with untreated curves usually have more back pain $[2,5]$, loss of function, external deformity, poor self-image, and in more severe cases, can impair respiratory capacity later in their life. Bracing is typically prescribed either based on guidelines set by the Scoliosis Research Society [9] or by the Society on Scoliosis Orthopaedic and Rehabilitation Treatment (SOSORT) [10], in which the Cobb angle is greater than $20^{\circ}$ with considerable growth remaining or show at least $5^{\circ}$ of Cobb angle increase between consecutive clinic visits. Recent scientific evidence has shown that brace treatment is effective [11-14], and a pilot study from a single centre has shown a predicted success rates of $95 \%$, when brace wear quantity combined with the brace wear quality is over $43 \%$ of the prescribed dosage [15]. A combined value of brace wear quantity and quality can be achieved in many different ways by trading off wear time and wear tightness; a subject can wear the brace $43 \%$ of prescribed time $(9.9 \mathrm{~h} /$ day $)$ and $100 \%$ of time at the prescribed tightness level. Similarly, when a subject wears a brace $100 \%$ of prescribed time ( $23 \mathrm{~h} /$ day), but only $43 \%$ of time at the prescribed level, the subject may get a similar result. Besides these two factors, the (a) growth or curve based risk and (b) the in-brace correction $[16,17]$ also affect brace treatment outcomes. The curve-based risk is estimated by physical maturity, gender, the severity and location of the curve, and the spinal balance. The in-brace correction may be affected by the brace design and spinal flexibility.

A typical spinal brace is a hard plastic shell with pads installed inside the liner to concentrate and direct the corrective pressure to oppose the spinal curvature. However, the locations of pads are set empirically based on guidelines for the type of the brace or knowledge derived from orthotists' experiences. Suboptimal pad placement and applied pressure will reduce the in-brace correction which is typically reviewed 6 weeks after the brace has been initiated. If the in-brace correction is not deemed to be satisfactory by the treating orthopedic surgeon, the patient returns to the orthotist for readjustment. This adjustment is required because there is no real time feedback provided to the orthotist during the brace design and construction stage. The standard of care requires the use of radiographs to check the in-brace correction. Radiographs are not taken during brace design and construction to minimize radiation exposure to growing children because of the increased risk of cancer. Unfortunately, after the adjustment, the in-brace correction examination is often required again which increases cumulative radiation exposure and shortens effective brace usage.

Although finite element (FE) models have been developed to determine optimal orientations and load magnitudes of pressure pads for brace design $[18,19]$, these still have practical limitations [20] with evaluation of the brace correction not available until the in-brace followup clinic. Recently, ultrasound (US) imaging, a real-time non-invasive and non-ionizing method, was demonstrated to be successful in measuring proxy Cobb angles, vertebral rotation, and flexibility [21-27]. The proxy Cobb angles which use vertebrae lamina positions rather than end plates, measured from ultrasound images have high intra- and inter-reliability as well as correlate well with radiographic measurements [22, 26]. Furthermore, there were studies applying ultrasound to determine the optimum location of the major brace pad [28, 29], but their approach did not provide real-time feedback nor determine the optimum pad pressure. Their ultrasound data were processed between the time the patient had their brace fitting and were returned to receive the modified brace. Researchers were also able to use ultrasound to investigate the time lag between application of spinal orthosis and its effect on scoliotic curvature [30]. Therefore, a clinical trial using ultrasound to assist orthotists to determine optimum pad pressure level and location during the brace design stage was conducted. This paper reports the immediate results obtained from this clinical trial.

\section{Methods \\ Patients}

Seventeen consecutive AIS subjects ( 2 males, 15 females; age $13.2 \pm 1.5$ years, Cobb $32 \pm 9^{\circ}$ ), with retrospectively collected data who were prescribed a new full time TLSO between January and June 2013 and met the inclusion criteria, served as the control group to match the intervention group recruitment. The distribution of the primary curve of the control group was 7 major thoracic, 6 thoracolumbar, and 4 lumbar curves. Another 17 new AIS subjects (2 male, 15 female; age $13.2 \pm$ 1.4 years, Cobb $35 \pm 8^{\circ}$ ), who were prescribed a TLSO were prospectively recruited between January 2014 and April 2015 into the intervention group. There was no significant difference of the Cobb angle between groups. The distribution of the primary curve of the intervention group was 6 major thoracic, 6 thoracolumbar, and 5 lumbar curves. Local ethics approval (Pro00028133) was granted by the local institution ethics board and all subjects signed consent forms before participation. The inclusion criteria followed the guidelines set by the 
non-operational management committee of the Scoliosis Research Society [9] (a) age 10 years or older when brace is prescribed, (b) Risser 0-2, (c) primary curve angles $20^{\circ}-45^{\circ}$, (d) no prior treatment, and (e) if female, either pre-menarchal or less than 1 year post-menarchal. Both participating orthotists are aligned with the same pediatric scoliosis program and worked together using the same methodology to design spinal brace for over 10 years. There was no change on the X-ray system and the clinical protocol during the entire recruitment period (January 2013-April 2015).

\section{Control group protocol}

For the control group, the traditional plaster cast and molded method with the assistance of the Providence brace system to design spinal braces was used. The orthotist first reviewed the standing posteranterior prebrace radiograph to identify the location of the apices. $\mathrm{He} /$ she then applied a plaster rigid wrap to the AIS body while the subject is standing and instructed the subject to lay upon the Providence brace system. The orthotist used the bolsters to apply pressures and adjusted the pressure level based on the location of the curve apex and his/her experience. After the plaster hardened, the subject stood up again to remove the hardened cast. Reflective markers were then placed around the cast and then scanned by a handheld laser scanner to create a 3D casting image file. The 3D file was then imported into software that was linked to a carving machine. Some minor adjustment was done at this stage to smooth the surface. A 3D body mold was then carved using foam material. After subjective modifications for improved fitting and comfort on the foam positive mold, a brace was fabricated. Subjects typically returned to the orthotist to fit the brace and make the final adjustments within a week. After that, the subject would use the brace for about 6 weeks, slowly building up their wear time, and returned to the scoliosis clinic to evaluate the design of the brace primarily based on wearability and the correction obtained from the in-brace radiograph.

\section{Intervention group protocol}

A custom Providence brace standing frame, a medical ultrasound (US) system, a custom pressure measurement system, and in-house US measurement software were used to assist brace casting for the intervention group. A $14 \mathrm{~cm} \times 50 \mathrm{~cm}$ opening was cut at the middle of the Providence frame to allow for the ultrasound scanning probe. Figure 1 shows the back of the frame and the custom Providence brace design set up with a subject. The subject wore a gown and stood against the standing frame. An operator with several years US scanning experience scanned the subject using the US system. It took approximately $1.5 \mathrm{~min}$ to acquire, process, and display the image. The pre-brace X-ray and the standing pre-pressure US spinal image were displayed side-byside to assist the orthotist to decide on pressure pads locations. The orthotist used the custom standing Providence brace design system to secure bolsters with subjectively determined applied pressure levels against the patient's torso to simulate in-brace correction. At each bolster, an air bag was attached on the surface to measure the interface pressure applied between the bolster and body. The simulated in-brace US scan was then acquired. A real-time US spinal image was displayed and the proxy Cobb angles were measured using in-house developed software. This process took less than $2 \mathrm{~min}$. The difference of the ultrasound measurements compared to the corresponding radiographic measurements was $2-3^{\circ}$ with good consistency [22]. The orthotist then

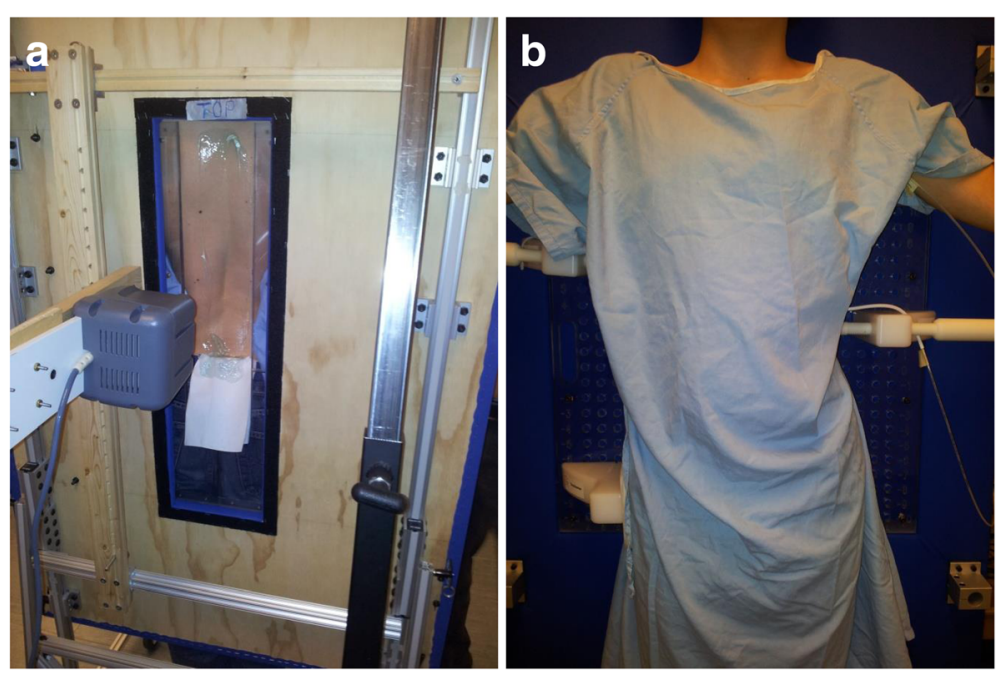

Fig. 1 a The opening at the back of the frame, and $\mathbf{b}$ a subject stands on a frame with a custom Providence brace design system 
decided if altering bolster locations and pressure levels might improve correction. Another US scan was taken if the bolster positions were altered. The procedures were repeated until the orthotist attained the best simulated in-brace correction configuration. The target goal was still to try to get at least $50 \%$ correction. During scanning, the pressure levels at each bolster were recorded. Figure 2 shows (a) the pre-brace standing X-ray with a right thoracic curve of $37^{\circ}$ between $\mathrm{T} 8$ and T12, (b) the standing baseline US image with proxy Cobb angle $35^{\circ}$, (c) the first US scan with axilla, thoracic, and lumbar pads pressure levels at 60,75 , and $75 \mathrm{mmHg}$, respectively, at which the Cobb angle is $25^{\circ}$, (d) the second US scan with axilla, thoracic, and lumbar pads pressure levels at 60, 90, and $90 \mathrm{mmHg}$, respectively at which the Cobb angle was $23^{\circ}$. The location of each bolster relative to the waist level was recorded. The orthotist then applied a plaster rigid wrap and identical pressure levels to the subject to the best stimulated in-brace correction configuration on a supine position with the Providence system. The pads' positions and pressure levels recorded from the standing frame were applied. After the plaster hardened and was removed, the cast was scanned by a handheld laser scanner to create a positive mold which was used for brace fabrication. Figure 3 shows the US second trial image overlapped with the in-brace radiograph at which the Cobb angle from the in-brace radiograph was $21^{\circ}$.

\section{First follow-up clinic}

Approximately, 6 weeks after braces initiation, all subjects returned to scoliosis clinics to inspect the effectiveness of the brace based on the in-brace correction. The treating orthopedic surgeons used the target threshold of in-brace Cobb correction of $50 \%$. They also used their clinical experience to consider whether the in-brace correction was optimal because the target threshold may not be attainable for rigid curves. If the surgeon was not satisfied with the in-brace correction, the subject would return to the orthotist for adjustments. Ultrasound was not used to assist in the adjustment for either group. Additional follow-up clinic visits with radiographs occurred approximately 2 months after adjustments.

\section{Results}

In the 17 control subjects, the major pre-brace Cobb angle was $32^{\circ} \pm 9^{\circ}$. Eight of these required brace adjustment (47\%) and 3 of these adjusted subjects (38\%) requiring a second adjustment. A total of 11 brace adjustments were needed and 28 in-brace radiographs were taken (average 1.6 radiographs per subject). The average in-brace major Cobb angle correction at the first in-brace follow-up clinic and at the final accepted follow-up clinic were $33 \pm 19 \%$ and $40 \pm 20 \%$, respectively.

For the intervention group, the major pre-brace Cobb angle from the radiographs prior to bracing was $35^{\circ} \pm$ $8^{\circ}$. Only 1 subject $(6 \%)$ required adjustment. A total of 18 in-brace radiographs were taken (average 1.1 radiographs per subject). The orthotist was satisfied with the first attempt with the US information in 8 out of 17 cases. With 9 subjects, the location and pressure level of the bolsters were altered one time. Among these 9

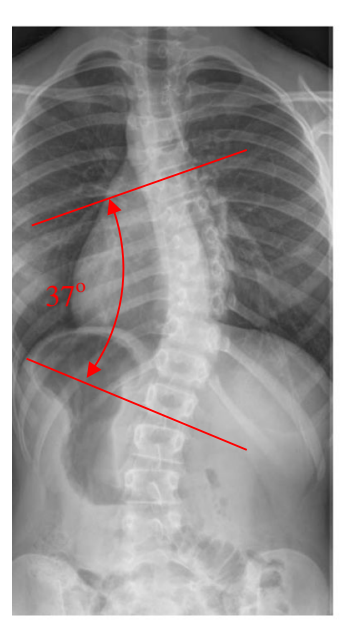

(a)

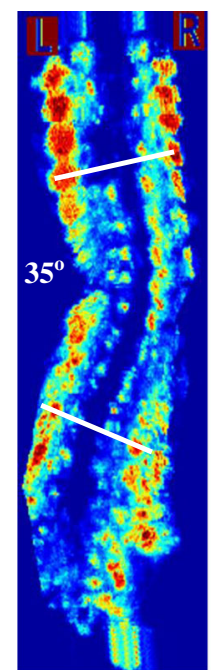

(b)

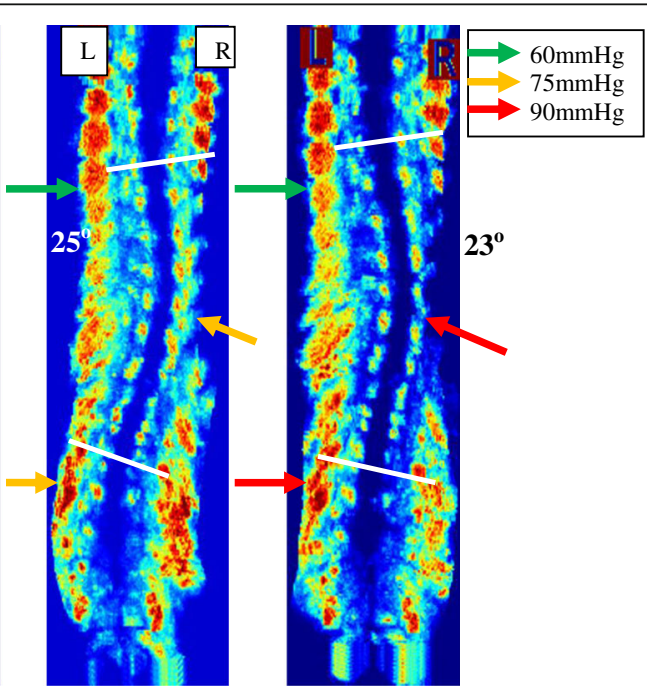

(c) (d)

Fig. 2 a The standing pre-brace X-ray with Cobb angle $37^{\circ}$. b The baseline US scan (Cobb angle $35^{\circ}$ ). c The first trial US scan $\left(\right.$ Cobb angle $25^{\circ}$ ). d The 2nd trial US scan (Cobb angle 23) 


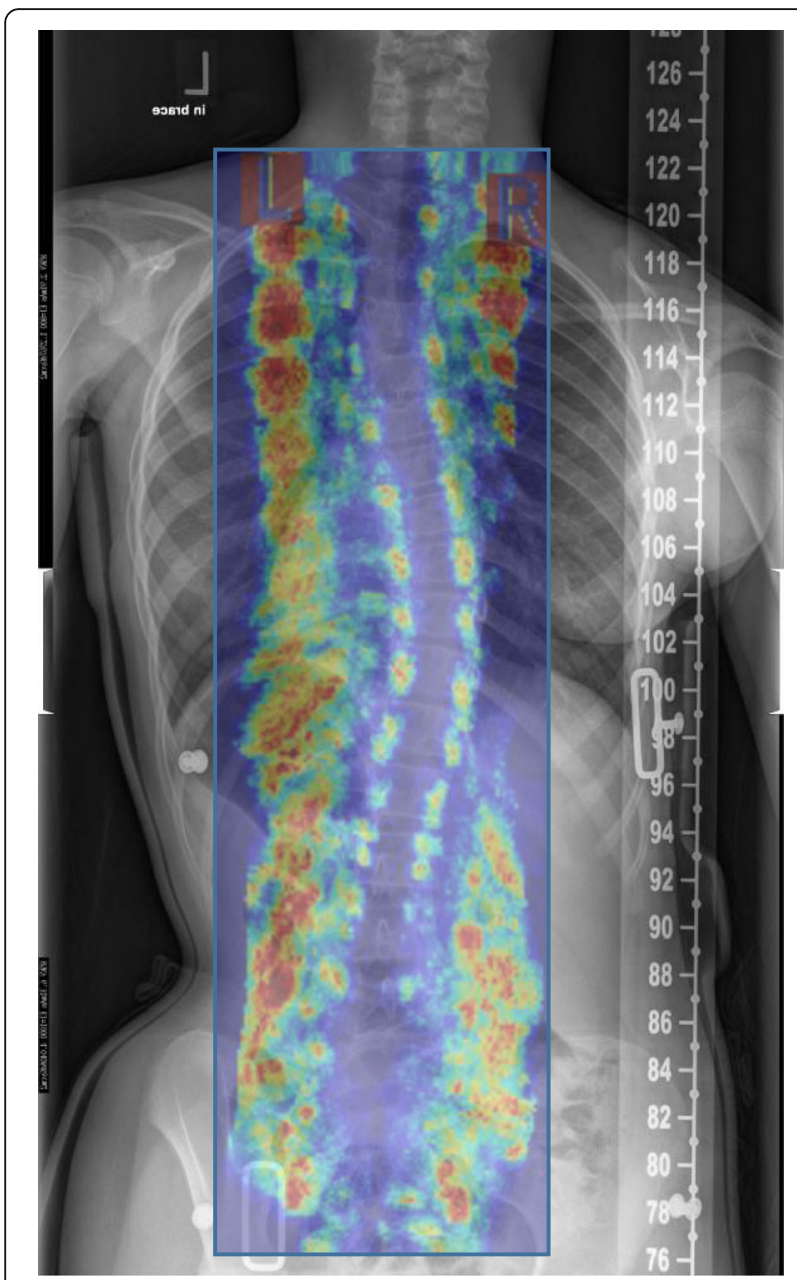

Fig. 3 The second US trial overlapped with the in-brace radiograph in which the Cobb angle from the radiograph was $21^{\circ}$

revised cases, 7 showed better stimulated in-brace corrections, 1 had no change, and 1 got worse. The intervention resulted in 7 out of 17 subjects (42\%) having their brace designed using an improved pressure level and/or pad placement. For the 7 improved cases, the inbrace Cobb correction from the US measurements in the first and second trials were $29 \pm 11 \%$ and $42 \pm 14 \%$, respectively. For the intervention group as a whole, the average final in-brace Cobb angle was $19^{\circ} \pm 8^{\circ}$ which was $48 \pm 17 \%$ in-brace correction, which was slightly higher than the simulated US in-brace correction.
The $p$ value of the chi-square for requiring brace adjustment between the control and the intervention groups was 0.0065 which was a statistically significant difference. The $p$ values of the unpaired two sided Student's $t$ test of the in-brace correction between the two groups were 0.02 and 0.22 between the first and second time of adjustment, respectively. It showed statistically significant difference between the US and the first time for the control group, but no statistically significant difference between the US and the second time of the control group. The reduction of the number of in-brace radiographs was large, 18 in-brace radiographs from the US group versus 28 in-brace radiographs from the control group, a saving of 10 radiographs in 17 subjects. Table 1 also shows the comparison of the health system time to cast and make the brace adjustment between the control and the intervention groups; on average an extra $1 \mathrm{~h} /$ per subject was needed in the control group. Furthermore, the time that the control and the intervention group received their optimum designed brace after prescription averaged $3.5 \pm 1.9$ months compared to $2.1 \pm$ 0.5 months. There was a significant delay to start the effective brace treatment between the two groups.

\section{Discussion}

Brace treatment is now generally accepted as a proven effective method to stop the progression of AIS. Besides compliance, a good brace design is vitally important. In current practice, the skill and experience of the orthotist are the major factors which affect the design of the brace. The pressure pads' levels, locations, and directions are subjectively selected by the orthotist. Without real-time feedback, trial and error in brace design is used. Lack of acceptable in-brace correction may trigger brace adjustment. Even though $\mathrm{Li}$ et al. [28, 29] applied the ultrasound method to assist brace fitting by investigating the locations of pressure pads, they did not provide the real-time feedback to the orthotist. They processed the data later to determine the optimum pad location and required patients to have an extra visit to receive the final brace. In this study, the intervention group has $7 / 17$ (42\%) that benefitted from having a brace adjustment after the initial setting of the pad placements. Those 7 cases which included 3 thoracic, 2 thoracolumbar, and 2 lumbar cases, did not indicate this

Table 1 Comparison of the casting and the brace adjustment time per subject

\begin{tabular}{|c|c|c|}
\hline & Control group & Intervention group \\
\hline Casting time & $17 \mathrm{~h}$ (1 h per subject) & $20.4 \mathrm{~h}$ (1.2 h per subject) \\
\hline Brace adjustment time & $11 \mathrm{~h}$ (1 h per adjustment) & $1 \mathrm{~h}$ \\
\hline Extra scoliosis clinic & $11 \mathrm{~h}$ & $1 \mathrm{~h}$ \\
\hline Total time & $39 \mathrm{~h}$ & $22.4 \mathrm{~h}$ \\
\hline Health system time per subject & $2.3 \mathrm{~h}$ & $1.3 \mathrm{~h}$ \\
\hline
\end{tabular}


method was only beneficial for specific types of curves. However, since the number of cases is still limited, no conclusive statement can be made. The advantage with the intervention group was that the adjustment was made prior to brace fabrication rather than after the first follow-up visit. The compromise between the comfort and treatment outcomes is influenced by how aggressively the orthotist designs the brace. With the immediate feedback, 7 out of 9 cases $(80 \%)$ showed the revised bolster placements or pressure alterations resulted in better correction than the first trial. This demonstrates how importantly the pressure pads location affects the effectiveness of the brace treatment. The subjects are able to report their pressure tolerance level that they feel in real-time. Requiring brace adjustment increases not only the number of radiographs and the cost of the health care system (orthotists', surgeons' and clinics time), but also the burden for the families that they need to travel to both brace adjustment and extra follow-up clinics. Furthermore, the benefits of getting the best designed brace in the shortest time may improve the overall effectiveness of the brace treatment because the patient will be using the brace most effectively sooner, during the most beneficial period of rapid adolescent growth. More clinical data are required to truly answer the total benefits of using ultrasound to assist brace casting. The limitation of this method is an experienced ultrasound technician is required during the brace casting to acquire and analyze the data. To overcome this, an automatic ultrasound machine which can scan the back automatically is being considered for future improvements. Also, the custom software developed for the ultrasound imaging measurement needs to be enhanced so that $3 \mathrm{D}$ information and automatic measurements can be obtained without requiring significant operator experience.

\section{Conclusions}

The use of the ultrasound system provided a radiationfree method to determine the optimum pressure level and location to obtain the best stimulated in-brace correction during brace casting. Although the long-term results have not yet known the immediate benefits of reduced cost, radiation exposure, and patient impact have merit. The number of radiograph taken per subject was reduced, and the acceptable in-brace correction was attained sooner in the intervention group with less burden on the families and patients.

\section{Abbreviations}

3D: Three dimensional; AIS: Adolescent idiopathic scoliosis; F: Female; M: Male; T: Thoracic; TLSO: Thoraco-lumbo-sacral orthosis; US: Ultrasound

\section{Funding}

Edmond Lou, Doug Hill, Douglas Hedden, and Marc Moreau received funding from the Glenrose Rehabilitation Hospital Foundation. Edmond Lou also received the grant from the Natural Sciences and Engineering Council of Canada (RGPIN-2015-04176).

\section{Availability of data and materials}

The datasets generated during and/or analyzed during the current study are available from the corresponding author on reasonable request.

\section{Authors' contributions}

EL participated in the conception, design and coordination, and to analysis and interpretation of data and prepared the manuscript. DLH is involved in the conception, design and coordination, and editing the manuscript. AD conceived of the study and participated to the acquisition of data. MT conceived of the study and participated to the acquisition of data. DH and MM were the attending orthopedic surgeons for the study; they are also involved in the conception, design, and coordination. All authors read and approved the final manuscript.

\section{Ethics approval and consent to participate}

All procedures performed in this pilot study involving human participants were in accordance with the ethics standards of The Human Research Ethics Board at University of Alberta. The project number of this ethics approval is Pro00028133. Informed consent was obtained from all individual participants included in this study and their parents.

\section{Consent for publication}

Consent for publication was obtained from all participants and their parents.

\section{Competing interests}

The authors declare that they have no competing interests.

\section{Publisher's Note}

Springer Nature remains neutral with regard to jurisdictional claims in published maps and institutional affiliations.

\section{Author details}

1Department of Surgery, University of Alberta, 6-110F, Clinical Science Building, 8440-112 Street, Edmonton, Alberta T6G 2B7, Canada. Department of Research and Innovation Development, Glenrose Rehabilitation Hospital, Edmonton, Alberta T5G 0B7, Canada. ${ }^{3}$ Department of Prosthetics and Orthotics, Glenrose Rehabilitation Hospital, Edmonton, Alberta T5G 0B7, Canada.

Received: 16 December 2016 Accepted: 10 July 2017 Published online: 08 August 2017

\section{References}

1. Lonstein JE, Carlson JM. The Prediction of curve progression in untreated idiopathic scoliosis during growth. J Bone Joint Surg. 1984;66A:1061-71.

2. Lonstein JE. Adolescent Idiopathic Scoliosis. Lancet. 1994;344:1407-12.

3. James JIP. Idiopathic Scoliosis. The prognoses, diagnosis, and operative indications related to curve patterns and the age at onset. J Bone Joint Surg. 1954:36B:36-49.

4. Nachemson A. A long term follow-up study of non-treated scoliosis. Acta Orthop Scand. 1968;39(4):446-76.

5. Weinstein SL, Zavala DC, Ponseti IV. Idiopathic scoliosis: Iong term follow-up and prognosis in untreated patients. J Bone Joint Surg. 1981;63-A(5):702-12.

6. Weinstein SL, Ponseti IV. Curve progression in idiopathic scoliosis. J Bone Joint Surg. 1983;65-A:447-55.

7. Edgar MA, Mehta MH. Long-term follow-up of fused and unfused idiopathic scoliosis. J Bone Joint Surg. 1988;70(5):712-6.

8. Dickson JH, Mirkovic S, Noble PC, Nalty T, Erwin RW. Results of operative treatment of idiopathic scoliosis in adults. J Bone Joint Surg. 1995;77(4):513-23.

9. Richards BS, Bernstein RM, D'Amato CR, Thompson GH. Standardization of criteria for adolescent idiopathic scoliosis brace studies: SRS Committee on Bracing and Nonoperative Management. Spine. 2005;30:2068-75.

10. Stefano N, Aulisa AG, Lorenzo A, et al. 2011 SOSORT guidelines: Orthopaedic and Rehabilitation treatment of idiopathic scoliosis during growth. Scoliosis. 2012;7:3. 
11. Dolan LA, Wright JG, Weinstein SL. Effects of bracing in adolescents with idiopathic scoliosis. N Engl J Med. 2014;370(7):681. Feb 13.

12. Aulisa AG, Guzzanti V, Galli M, Perisano C, Falciglia F, Aulisa L. Treatment of thoraco-lumbar curves in adolescent females affected by idiopathic scoliosis with a progressive action short brace (PASB): assessment of results according to the SRS committee on bracing and nonoperative management standardization criteria. Scoliosis. 2009;4:21.

13. Negrini S, Minozzi S, Bettany-Saltikov J, Zaina F, Chockalingam N, Grivas TB, Kotwicki T, Maruyama T, Romano M, Vasiliadis ES. Braces for idiopathic scoliosis in adolescents. Spine (Phila Pa 1976). 2010;35:1285-93.

14. Aulisa AG, Guzzanti V, Perisano C, Marzetti E, Falciglia F, Aulisa L. Treatment of lumbar curves in scoliotic adolescent females with progressive action short brace: a case series based on the Scoliosis Research Society Committee Criteria. Spine (Phila Pa 1976). 2012;37(13):E786-91.

15. Lou E, Moreau M, Mahood JK, Hedden DM, Hill D, Raso JV. How Quantity and Quality of Brace Wear Affect the Brace Treatment Outcomes for AIS Patients. J Eur Spine. 2016;25(2):495-9. doi:10.1007/s00586-015-4233-22015.

16. Lou E, Hill DL, Parent E, Raso VJ, Moreau MJ, Mahood JK, Hedden D. Prediction of Brace Treatment Outcomes. In: Proceeding of SOSORT 2009 The 6th International Conference on Conservative Management of Spinal Deformities. 2009. p. 2192.

17. Lou E, Chan A, Donauer A, Tilburn M, Hill D. Ultrasound assisted brace casting for adolescent idiopathic scoliosis, IRSSD Best Research Paper 2014 Scoliosis. 2015;10:13. doi:10.1186/s13013-015-0037-8.

18. Cheng FH, Shih SL, Chou WK, et al. Finite element analysis of the scoliotic spine under different loading conditions. Biomed Mater Eng. 2010;20:251-9.

19. Clin J, Aubin CE, Parent S, Sangole A, et al. Correlation between immediate in-brace correction and biomechanical effectiveness of brace treatment in adolescent idiopathic scoliosis. Spine. 2010;35(18):1706-13.

20. Clin J, Aubin CE, Parent S, Sangole A, Labelle H. Comparison of the biomechanical 3D efficiency of different brace designs for the treatment of scoliosis using a finite element model. Eur Spine J. 2010;19:1169-78.

21. Chen W, Lou E, Le LH. Reliability of the axial vertebral rotation measurements of adolescent idiopathic scoliosis using the center of lamina method on ultrasound images: in-vitro and in-vivo study. J Eur Spine. 2016. doi: 10.1007/s00586-016-4492-6.

22. Zheng R, Young M, Hill D, Le L, Moreau M, Mahood JK, Hedden D, Southon S, Lou E. Improvement on the Accuracy and Reliability of Ultrasound Coronal Curvature Measurement on AIS with the Aid of Previous Radiographs. Spine. 2016;41(5):404-11. doi:10.1097/BRS.0000000000001244.

23. Wang Q, Li M, Lou E, Wong MS. Reliability and Validity Study of Clinical Ultrasound Imaging on Lateral Curvature of Adolescent Idiopathic Scoliosis. PLOS ONE. 2015. doi:10.1371/journal.pone.0135264.

24. Young M, Hill D, Zheng R, Lou E. Reliability and Accuracy of Ultrasound Measurements With and Without the Aid of Previous Radiographs in Adolescent Idiopathic Scoliosis. J Eur Spine. 2015;24(7):1427-33. doi:10.1007/s00586-015-3855-8.

25. Zheng R, Chan A, Chen W, Hill D, Le LH, Moreau M, Mahood JK, Hedden D, Southon S, Lou E. Intra- and Inter-rater Reliability of coronal curvature measurement for AlS using ultrasonic imaging method. J Spine Deform. 2015;3(2):151-8. doi:10.1016/j.jspd.2014.08.008.

26. Lou E, Zhang R, Donauer A, Tilburn M, Hill D, Raso J. Can ultrasound imaging be used to determine curve flexibility when designing spinal orthoses? Scoliosis Spinal Disord. 2016;11(Suppl 1):23. Published online 2016 Aug 23. doi:10.1186/s13013-016-0077-8.

27. Zheng YP, Lee T, Lai K, Yin B, Zhou GQ, Jiang WW, Cheung J, Wong MS, $\mathrm{Ng} \mathrm{B}$, Cheng J, Lam TP. A reliability and validity study for Scolioscan: a radiation-free scoliosis assessment system using 3D ultrasound imaging. Scoliosis Spinal Disord. 2016:11:13

28. Li M, Cheng J, Ying M, Ng B, Zheng YP, Lam TP, Wong WY, Wong MS. Application of 3-D ultrasound in assisting the fitting procedure of spinal orthosis to patients with adolescent idiopathic scoliosis. Stud Health Technol Inform. 2010;158:34-7.

29. Li M, Cheng J, Ng KW, Lam TP, Zheng YP, Ying M, Wong MS. Could clinical ultrasound improve the fitting of spinal orthosis for the patients with AIS? Eur Spine J. 2012;21:1926-35.

30. Li M, Wong MS, Luk KD, Wong KW, Cheung KM. Time-dependent response of scoliotic curvature to orthotic intervention: when should a radiograph be obtained after putting on or taking off a spinal orthosis. Spine. 2014;39:1408-16.

\section{Submit your next manuscript to BioMed Central and we will help you at every step:}

- We accept pre-submission inquiries

- Our selector tool helps you to find the most relevant journal

- We provide round the clock customer support

- Convenient online submission

- Thorough peer review

- Inclusion in PubMed and all major indexing services

- Maximum visibility for your research

Submit your manuscript at www.biomedcentral.com/submit

Biomed Central 